\title{
Ein Recht auf Gesundheit
}

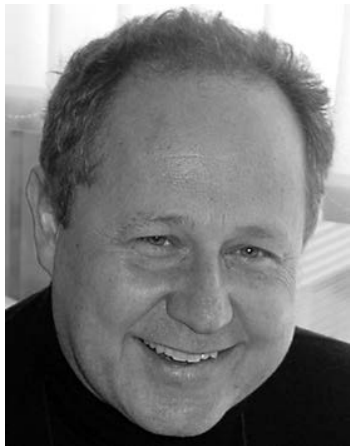

Prof. Dr. med. Manfred Wildner
Bibliografie

DOI http://dx.doi.org/

10.1055/s-0042-121549

Gesundheitswesen 2016;

78: 799-800

(c) Georg Thieme Verlag KG

Stuttgart · New York

ISSN 0941-3790

Korrespondenzadresse

Prof. Dr. med. Manfred Wildner

Bayerisches Landesamt für Gesundheit und Lebensmittelsicherheit

Veterinärstraße 2

85764 Oberschleißheim

manfred.wildner@lgl.bayern.de
Mose muss rot vor Zorn - und Anstrengung - gewesen sein, als er die Gesetzestafeln 1.0 angesichts des Verhaltens seines Volkes auf der langen Wanderung durch die Sinai-Wüste zerschlug. Die sittlich ausgerichtete Hälfte der dort eingemeiBelten Gebote ist ohnehin eine kulturübergreifende, universale Menschheitsleistung: Nicht töten, nicht stehlen, kein sexuelles Fehlverhalten, keine falsche Rede und - ein Novum gegenüber dem jüdischen Dekalog - Abstinenz gegenüber Rauschmitteln finden sich als Anspruch auch in den asiatischen Weisheitsreligionen des Buddhismus und Taoismus. Als Version 2.0 fanden Moses Steintafeln dann doch noch ihren Weg in die - wohlverschlossene - Bundeslade. Weitere Gesetze folgten. Die Verkündigung oblag den Priestern, wie auch die weitere Verschriftlichung im Rahmen der Tora-Redaktion (ca. 950-550 v.Chr.). Im gebildeten Athen setzte man(n) frühzeitig auf öffentlich zugängliche Holztafeln im Drehgestell und eine zeitlich begrenzte Geltungsfrist. Im römischen Staat musste, beeinflusst von der griechischen Gesetzgebung, die Verschriftlichung und Veröffentlichung von Gesetzen den Priestern und Patriziern vom Volk mühsam abgerungen werden - im Ergebnis waren es 12 öffentlich aufgestellte Bronzetafeln. Die Realisierung des Menschheitstraums von Fairness durch nachvollziehbare Rechtsanwendung musste offensichtlich erkämpft werden, „Transparency International" ist nur eine moderne Aktualisierung dieser Anstrengung.

Universal gültige, unteilbare und unveräußerliche Menschenrechte sind ein anderer großer Menschheitstraum. Wie wohl die meisten dieser Träume ist er aus Not und Leid geboren. 1948, nach der Katastrophe der Weltkriege des 20. Jahrhunderts, verabschiedete die Vollversammlung der Vereinten Nationen eine allgemeine Erklärung der Menschenrechte - diesmal gebunden und auf Papier. In deren Artikel 25 findet auch ein „Menschenrecht auf Gesundheit“, wie schon zuvor im Verfassungsentwurf der Weltgesundheitsorganisation (WHO) von 1946, einen ersten Ausdruck: ,Jeder hat das Recht auf einen Lebensstandard, der seine und seiner Familie Gesundheit und Wohl gewährleistet, einschließlich Nahrung, Kleidung, ärztliche Versorgung und notwendige soziale Leistungen...". Dieser Traum hat sich in weiteren Erklärungen entfaltet, wie den Erklärungen zu den Rechten des Kindes von 1959 und insbesondere auch in Artikel 12 des internationalen Paktes über wirtschaftliche, soziale und kulturelle Rechte (UN Sozialpakt, 1966): „Die Vertragsstaaten erkennen das Recht eines jeden auf das für ihn erreichbare Höchstmaß an körperlicher und geistiger Gesundheit an".

Ein Recht auf Gesundheit? Ist das nicht einmal mehr eine unerreichbare Utopie angesichts der auch biologisch ungleich verteilten Gesundheitschancen, welche oft noch einmal durch soziale Ungleichheiten in ihren Auswirkungen verstärkt werden? Und ist hier die Rede von Gesundheit in der Definition der WHO als „Zustand vollständigen körperlichen, seelischen und sozialen Wohlbefindens" [1]? Oder in der lebensnäheren Definition des „Health for All“-Programms der WHO von 1981, welches Gesundheit als Befähigung zu einem „sozial und ökonomisch produktiven Leben“ versteht [2]? Papier ist geduldig, doch auch Menschheitsträume haben eine große zeitliche Reichweite. Die Idee eines Menschenrechtes auf Gesundheit wurde in Zusammenhang mit der weltweiten AIDS-Kampagne der Vereinten Nationen weiter vertieft und ist international mit der Person Jonathan Manns eng verknüpft $[3,4]$. Von der Bundesärztekammer wurde die Thematik aufgegriffen und in den 1990-iger Jahren das Amt des Menschenrechtsbeauftragten geschaffen. Der Zusammenhang von Recht und Gesundheit wurde in Deutschland auch unter dem Aspekt der Bürgerbeteiligung im Gesundheitswesen sowie im Zusammenhang mit Patientenrechten weiter diskutiert [5-7]. 2013 wurde ein Patientenrechtegesetz verabschiedet, welches insbesondere Behandlungsfehler, Arzthaftung, Aufklärung, die Einsicht in die Krankenakten, die Beweislastrechte gegenüber den Krankenkassen, die Patientenbeteiligung, die Patienteninformation und den Aspekt der Patientenverfügung regelt (www.pa tienten-rechte-gesetz.de, Download 02.11.2016). Menschen müssen das Recht und damit ihre Rechte kennen, um sie wahrnehmen zu können: Hier schafft ein zusammenführendes Gesetz die Klarheit, welche verstreute juristische Quellen in unterschiedlichen Gesetzen nicht schaffen können. Gostomzyk und von Mittelstaedt weisen auf die auch in Deutschland liegenden Wurzeln eines Rechtes auf Gesundheit hin [8]. Bereits von Salomon Neumann wurde 1874, im preußischen „Vormärz“ 100 Jahre vor der Erklärung der Menschenrechte, die Forderung einer Umsetzung liberaler und demokratischer Ideen auch in Bezug auf Gesundheit erhoben [9]: Er zieht eine Parallele zwischen einem Recht auf Gesundheit und dem im preußischen Staat unangefochtenen Recht auf Eigentum. Der Ansatz Salomon Neumanns fokussiert zunächst auf ein Abwehrrecht bezogen auf die Gesundheit. Dieser Ansatz findet allgemein leichter Zustimmung als das in der 
UN-Sozial-Charta zusätzlich formulierte Anspruchsrecht auf gesundheitliche Versorgung. Diese Unterscheidung hat, insbesondere nach Ende des Kalten Krieges, zu einer Differenzierung innerhalb der Menschenrechtsbewegung geführt, mit einer Betonung der Unterscheidung zwischen bürgerlichen und politischen Rechten einerseits und sozialen und ökonomischen Rechten andererseits. Bezogen auf die gesundheitswissenschaftliche Forschung wurden im Zusammenhang mit Menschenrechten 3 Ansätze formuliert: Untersuchungen $\mathrm{zu}$ den Auswirkungen der (Gesundheit-)Politik auf die Menschenrechtssituation, Untersuchungen zu den Auswirkungen der Menschenrechtssituation auf die Gesundheit und zum dritten die weitergehende Erforschung dieser Interaktion zwischen Menschenrechten und Gesundheit $[3,4]$. Diesem Ansatz liegt ein umfassendes Gesundheitsverständnis zugrunde, wie es bspw. in der Ottawa-Charta zur Gesundheitsförderung vertreten wird oder auch in der Ljubljana-Charta on Reforming Health Care des europäischen Regionalbüros der Weltgesundheitsorganisation (1996, EUR/ICP/ CARE9401/CN01). Einflüsse ergeben sich aus der Verfügbarkeit, dem Zugang, der Akzeptanz und der Qualität der gesundheitlichen Versorgung, der einwirkenden sozialen, kulturellen und ökologischen Umweltfaktoren im Allgemeinen wie auch im Speziellen z.B. am Arbeitsplatz, aus der Verfügbarkeit von Information wie auch aus der Befähigung zum Verständnis von Gesundheitsinformation und von Gesundheit in ihren vielfältigen Bezügen (health literacy) $[10,11]$. Der Zusammenhang von sozialer Ungleichheit und Gesundheit ist gut dokumentiert (siehe auch [12]). Eine empirische Forschung zu den Zusammenhängen von Gesundheit und Menschenrechten bzw. zu Patientenrechten hat viele Aspekte: Die historische Forschung zu den Medizinverbrechen im Nationalsozialismus, der Diskurs um die Humanisierung der Arbeitswelt, z.B. mit empirischen Studien zur Qualität des Arbeitsschutzes in Abhängigkeit von der Qualität der Mitbestimmung im Betrieb, zum Stichwort Globalisierung auch die Studien zu Arzneimitteltests in der Dritten Welt usw. [13,14]. Neuen Auftrieb hat dieses Thema durch die Diskussion um die gesundheitliche Versorgung von geflüchteten und asylsuchenden Menschen innerhalb der Europäischen Union und im Besonderen auch in Deutschland bekommen (siehe z.B. www.gesundheitein-menschenrecht.de). Und so werden neu die alten Fragen gestellt, nach der Würde des Menschen, nach der Einklagbarkeit von grundsätzlich zugestandenen Menschenrechten, nach Chancengleichheit, Gleichbehandlung und Mitgestaltung. Und dies nicht nur auf Ebene des Individuums und seiner möglichen Rolle als Koproduzent von Gesundheit, sondern auch bezogen auf die in kollektiver Verantwortung liegenden Regularien und Ausgestaltungen des Gesundheitswesens. Dass diese Fragen nicht leicht zu beantworten sind, insbesondere hinsichtlich der auch ethischen Aspekte von Verteilungsgerechtigkeit und Leistungsfähigkeit, ist nicht zu übersehen und sollte als wichtiger und zur Diskussion über ein Recht auf Gesundheit gehöriger Teil geduldige Berücksichtigung finden.

Solchen Fragen und auch weiteren Fragen der Systemgestaltung gehen die Beiträge in diesem Heft nach: Zur Salomon-Neumann-Medaille 2016 in Ehrung der gelungenen Gesundheitsberichterstattung des Robert Koch-Instituts, zum Recht auf Gesundheit in den Schriften des großen Sozialmediziners Salomon Neumann, zur gesundheitlichen Versorgung von Geflüchteten, zu zahnmedizinethischen Kenntnissen in der Praxis, zu Barrieren in der Thematisierung des Tabak- und Alkoholkonsums Schwangerer, zum subjektiven Erleben ihrer Kolonisation von MRSA-Trägern/innen, zur Verständlichkeit der Texte von Krankenhaus-Qualitätsvergleichen, zu Erfolgsfaktoren für eine verbesserte haus- ärztliche Versorgung in Oberösterreich, zur Weiterentwicklung der Regulierung des Inverkehrbringens von Arzneimitteln und Medizinprodukten sowie zum „Storytelling“ im Gesundheitsjournalismus.

Papier ist geduldig - dieser Spruch wird oft zitiert, um den langen Weg von der Formulierung und schriftlichen Fixierung eines Rechtsanspruchs bis zu seiner tatsächlichen Umsetzung zu beschreiben. Von einem „langen Marsch durch die Institutionen“ träumte Rudi Dutschke in Erinnerung an den langen Marsch Mao Zedongs, im Rahmen der politisch geprägten studentischen Protestbewegung der 1968er Jahre, welche nun auch schon einige Zeit zurückliegt. Von bürgerlicher Seite wird wiederum ein maßvoller Umgang mit Utopien bei deren Umsetzung in gesellschaftliche Realität eingefordert: „Die Finanzautonomie und -schwäche des Staates sowie die Last des Bürgers, an der Bewahrung und Herstellung der Gesundheit eigenverantwortlich mitzuwirken, schützt vor Übertreibungen“ [15]. Vermutlich gehören alle diese Stimmen zu den notwendigen Diskursen auf dem langen Marsch der Menschheit und den auf diesem Weg Orientierung gebenden Utopien, welcher zwar 1847, 1948 oder 1968 markante Stationen erreicht haben mag, dabei in seinen Anfängen um vieles weiter zurückliegt. Noch eine Stimme aus der Vergangenheit? „Litterae non erubescunt - Buchstaben erröten nicht“ (Cicero 106-43 v. Chr.). Buchstaben mögen zwar nicht erröten, wir Menschen aber sollten dies schon tun, wenn wir einem Menschheitsanspruch auf Dauer nicht genügen.

\section{Literatur}

1 Preamble to the Constitution of the World Health Organization as adopted by the International Health Conference, New York, 19-22 June, 1946; signed on 22 July 1946 by the representatives of 61 States (Official Records of the World Health Organization, no. 2, p. 100) and entered into force on 7 April 1948

2 Mahler $H$. The meaning of Health For All by the year 2000. World Health Forum 1981; 2: 5-22

3 Mann J, Gostin L, Gruskin S, Brennan T, Lazzarini Z, Fineberg H. Health and human rights. Health Hum Rights 1994; 1: 6-23

4 Mann J. Human rights and the new public health. Health and human rights 1994; 1 : 229-233

5 Hart D. Bürgerbeteiligung im Gesundheitswesen: Rechtliche Analysen und Perspektiven. Public Health Forum 1999; 7: 7-8

6 Wildner M, Busse R, Brunner-Wildner A. Patientenpartizipation in Europa. Forschungsjournal neue soziale Bewegung 2002; 15: 106-113

7 Kranich C, Böcken J. Patientenrechte und Patientenunterstützung in Europa. Baden Baden: Nomos Verlagsgesellschaft; 1998

8 Gostomzyk JG, von Mittelstadt G. Salomon Neumann (1819-1908) und das Recht auf Gesundheit. Gesundheitswesen 2016; 78: 804-807

9 Neumann S. Die öffentliche Gesundheitspflege und das Eigenthum. Kritisches und Positives mit Bezug auf die preußische Medizinalverfassungs-Frage.Verlag Adolf Ries; Berlin: 1847, elektrischer Verlag Berlin 2014 (Hrsg. Günther Regneri).

10 Nutbeam D, Kickbusch I. Advancing health literacy: a global challenge for the $21^{\text {st }}$ century. Health Prom Int 2000; 15: 183-184

11 Nutbeam $D$. Health literacy as a public health goal: a challenge for contemporary health education and communication strategies into the $21^{\text {st }}$ century. Health Prom Int 2000; 15: 259-267

12 Wildner M, Brunner A, Fischer R, Ludwig MS. Menschenwürde und Chancengleichheit: Menschenrechte im gesundheitlichen Kontext. In: Mielck A, Bloomfield K, (Hrsg.). Soziale Epidemiologie. Eine Einführung in die Grundlagen, Ergebnisse und Umsetzungsmöglichkeiten. Juventa Verlag; Weilheim und München: 2001: 383-395

13 Frewer A, Bielefeldt H., (Hrsg.). Das Menschenrecht auf Gesundheit - Normative Grundlagen und aktuelle Diskurse. Bielefeld, Transkript-Verlag 2016

14 Wildner M, Fischer R, Brunner A. Development of a questionnaire for quantitative assessment in the field of health and human rights. Social Science and Medicine 2002; 55: 1725-1744

15 Pestalozzi C. Das Recht auf Gesundheit. Verfassungsrechtliche Dimension. Bundesgesundheitsblatt 2007; 50: 1113-1118 